\title{
Downlink Multi-User Massive MIMO Transmission using Receive Spatial Modulation
}

\author{
Ahmed Raafat, Member, IEEE, Adrian Agustin, Member, IEEE, and Josep Vidal, Member, IEEE
}

\begin{abstract}
In this paper, we consider the downlink of multi-user multiple-input-multiple-output system operating in the narrowband and outdoor of millimeter-wave propagation environment. First, we propose a receive spatial modulation (RSM) scheme based on energy efficient transceiver architecture for the single user system. We derive the RSM system assuming single radiofrequency (RF) chain at the user terminal (UT) and then, we propose a novel extension for multiple RF chains. At the UT, we consider analog switches to control which antennas are active during the transmission according to an optimization algorithm aimed at maximizing the spectral efficiency. At the base station (BS), we use analog switches to control the ON/OFF status of the RF chains based on an optimization algorithm to improve the energy efficiency at the BS. Then, we extend the proposed RSM scheme for multiple users. Specifically, we propose an algorithm to jointly optimize number of users, set of antennas and the power allocated for each user to maximize the weighted sum spectral efficiency. After that, for a given number of users and antennas per user, we formulate the power allocation problem as non convex optimization program. Hence, we propose alternating optimization algorithm to convert the non convex program into multiple convex problems. Finally, we compare the performance and the convergence of the proposed alternating optimization with successive convex approximation framework. Simulation results are presented to illustrate the single and multiple users system performance of the RSM scheme compared to conventional modulation transmission.
\end{abstract}

Index Terms-Receive spatial modulation, massive MIMO and millimeter-wave.

\section{INTRODUCTION}

$\mathbf{T}$ HE rapid growth of wireless industry opens the door for several use cases such as internet of things and device to device communications [1], [2] which require boosting the wireless network speed for attaining reliable communications. The vast spectrum available in millimeter-wave (mmWave) frequency band is one of the most promising candidates in achieving high speed communications [3], [4], [5]. However, the propagation of the radio signals at high carrier frequencies suffers from severe path-loss which is a bottleneck in increasing the coverage area [6]. Fortunately, the small wavelengths of the mmWave signals allow packing large number of antennas not only at the base station (BS) but also at the user terminal (UT) [7]. These massive antennas can be exploited in attaining high beamforming and combining gains to overcome the path-loss associated with the mmWave propagation. However, in conventional multiple-input-multiple-output (MIMO)

The authors are with the Department of Signal Theory and Communications, Universitat Politècnica de Catalunya, Barcelona 08034, Spain (e-mail: ahmed.raafat@upc.edu; adrian.agustin@upc.edu; josep.vidal@upc.edu).

The research leading to these results has been funded by the European Union's Horizon 2020 research and innovation programme under the Marie Sklodowzka-Curie grant agreement No 641985 . transceivers, each antenna is connected to specific radiofrequency $(\mathrm{RF})$ chain and high resolution analog-to-digitalconverter (ADC). Unfortunately, these devices are expensive and power hungry especially at mmWave frequency band.

Fully connected hybrid analog and digital precoding schemes [8], [9], [10] have been developed with the aim of replacing some of the power consuming RF chains by energy efficient analog devices. However, these architectures can be power consuming due to adding many analog devices with non negligible power consumption [11]. Partially connected hybrid architectures [12], [13], [14] have been proposed to improve the energy efficiency (EE) of the fully connected transceivers by reducing the number of analog devices. However, improving the EE entails a degradation in the spectral efficiency (SE).

Transmit spatial modulation (TSM) schemes have been developed with the aim of reducing number of RF chains at the transmitter (BS in the downlink (DL)) [15], [16]. In TSM, single antenna is active at the BS and the input data bits are divided into two parts. The first part is used to select the index of the active antenna while the second part is mapped into $M$-ary symbol that transmitted through the active antenna. Then, the UT applies the appropriate joint detector to the index of the active antenna and the $M$-ary symbol [17]. In generalized TSM schemes [18], [19], multiple antennas are activated according to the input data bits. However, the small number of active antennas at the BS reduces the beamforming gain and thus, TSM schemes become challenging in the DL transmission of the mmWave signals.

On the other hand, receive spatial modulation (RSM) schemes have been proposed for the indoor [20] and outdoor [21], [22], [23] of the DL mmWave propagation based on energy efficient UT architecture. In DL RSM, part of the input bits (spatial symbol) is used to activate set of UT antennas and the other part is mapped into $M$-ary modulation symbol. In [21], the authors exploited the definition of the RSM in attaining energy efficient UT design where the spatial symbol is detected using analog devices and the modulation symbol is detected by single RF chain. In [24], a DL multi-user RSM scheme has been proposed; however, a power consuming fully digital UT architecture is considered.

In this paper, we consider DL RSM scheme for multi-user MIMO system operating at mmWave outdoor and narrowband environment. The main novelty and contributions of this work are as follows

- We propose novel and energy efficient UT architecture that can work in two modes, RSM or conventional modulation (CM) through adjusting controlling switches. 
- For the sake of improving the SE, we extend the work in [21] into multiple RF chains at the UT and we propose novel combining scheme that achieves higher SE.

- We propose low complexity receive and transmit antenna selection algorithms aimed at maximizing the BS EE.

- We propose novel fast and efficient algorithm to jointly optimize number of users, set of antennas and the power allocated per user to maximize the weighted sum SE.

- At given set of users and antennas per user, we formulate the power allocation as non convex optimization problem. Then, we propose alternating optimization algorithm that convert the non convex program into multiple convex problems. Finally, we compare the performance and the convergence of the alternating optimization method with the successive convex approximation framework [25], [26].

- We present the simulation results to evaluate the proposed system performance and compare the RSM scheme with the $\mathrm{CM}$ transmission.

\section{SYSTEM MODEL}

We consider the downlink of mMIMO system operating at narrowband transmission of mmWave frequencies and outdoor environment. At first, we design the single user system and next we extend the design to multi-user system. The BS and each UT are equipped with $N_{t}$ and $N_{r}$ antennas, respectively.

\section{A. BS design}

As shown in Fig. 1a, we consider fully digital BS; however we can reduce the power consumption at the BS by deactivating set of RF chains based on the channel and we update this set every coherence time. We can control the ON/OFF status of the RF chains through $N_{t}$ control switches each connects/disconnects one antenna to RF chain and high resolution ADC as depicted in Fig. 1a. This architecture achieves high spectral efficiency (SE) under power consumption constrains as illustrated in Fig. 11a in [11]. In Sec. III-G, we illustrate how the antennas are selected at the BS where only $\left(N_{b} \leqslant N_{t}\right)$ transmit antennas are active and the rest are silent during the coherence interval.

\section{B. UT design}

We consider the energy efficient UT architecture proposed at [21] that consists of single RF chain, one high resolution ADC (power hungry devices) and $N_{r}$ ADs, $N_{r} 1$-bit ADCs (power efficient devices) as depicted in Fig. 1b. The AD measures the amplitude of the mmWave signal in the RF domain with negligible power consumption, high sensitivity and massive input impedance [27]. In RSM systems [28], deactivating the spatially correlated antennas at the UT reduces the effect of the spatial correlation among the UT antennas caused by the sparse propagation of mmWave signals in the outdoor environments and enhances the (SE) of RSM systems. In this work, we consider $N_{a} \leqslant N_{r}$ UT antennas are active during a transmission and we provide detailed analysis about the receive antenna selection (RAS) procedure in section $\mathrm{xx}$. In Fig. 1c, for the sake of improving the SE, we propose a novel energy efficient UT circuitry to extend the architecture in Fig. $1 b$ into multiple RF chains. In the proposed architecture, we consider energy efficient control switches. First, the switch $S_{k}^{\prime \prime}$ controls the ON/OFF status of the $k^{\text {th }}$ UT antenna. Second, only one of the switches $\left(S_{i 1}^{\prime}, \cdots, S_{i N_{r f}}^{\prime}\right)$ is ON to connect the $i^{t h}$ received symbol to a specific RF chain. Finally, the witch $S_{i j}$ can be always closed (independent combining scheme) or controlled by the received spatial symbol (dependent combining scheme) as illustrated in section xx. The switches status remains fixed within the channel coherence time.

\section{Transceiver circuitry power consumption}

We consider the electronic devices power consumption to evaluate the $\mathrm{EE}$ of the transceiver circuitry at the UT ( $\left.\mathrm{EE}_{\mathrm{UT}}\right)$ and at the $\mathrm{BS}\left(\mathrm{EE}_{\mathrm{BS}}\right)$. The power consumed by different devices can be modeled in terms of reference power $\left(P_{\text {ref }}\right)$ as follows [11], [29]

$$
\begin{gathered}
P_{\mathrm{LNA}}=P_{\mathrm{ref}}, P_{\mathrm{ADC}}=P_{\mathrm{DAC}}=\mathrm{FoM} \times f_{s} \times 2^{n}, \\
P_{\mathrm{RF}}=2 P_{\mathrm{ref}}, P_{\mathrm{SW}}=0.25 P_{\mathrm{ref}}, P_{\mathrm{PA}}=\left(\frac{1}{\eta}-1\right) P_{t}
\end{gathered}
$$

where $P_{\mathrm{LNA}}, P_{\mathrm{RF}}, P_{\mathrm{ADC}}, P_{\mathrm{SW}}$ and $P_{\mathrm{PA}}$ are the low noise amplifier, RF chain, analog-to-digital-converter, phase shifter, switch and power amplifier power consumption, respectively. The baseband power consumption $P_{\mathrm{BB}}=10 P_{\text {ref }}$ [11], the sampling frequency $f_{s}$ equals to twice the bandwidth, $n$ is the ADC quantization bits, the figure of merit FoM is technology dependent that takes $34.4 \mathrm{fJ} / \mathrm{Conv}$.-step at $n=12$ and $f_{s}=600 \mathrm{MS} / \mathrm{s}$ [30], the power amplifier efficiency $\eta$ reaches $40 \%$ at $28 \mathrm{GHz}$ [31] and $P_{t}$ is the transmit power from the BS. The AD consumes negligible amount of power. The power consumption and the EE of different circuitries can be expressed as

$$
\begin{gathered}
P_{C, \mathrm{BS}}=N_{b}\left(P_{\mathrm{RF}}+P_{\mathrm{DAC}}+P_{\mathrm{PA}}\right)+P_{\mathrm{BB}} \\
P_{C, \mathrm{UT}-\mathrm{SRF}}=N_{a}\left(3 P_{\mathrm{SW}}+P_{\mathrm{LNA}}\right)+P_{\mathrm{RF}}+P_{\mathrm{ADC}}+P_{\mathrm{BB}} \\
P_{C, \mathrm{UT}-\mathrm{MRF}}=N_{a}\left(3 P_{\mathrm{SW}}+P_{\mathrm{LNA}}\right)+N_{\mathrm{RF}}\left(P_{\mathrm{RF}}+P_{\mathrm{ADC}}\right)+P_{\mathrm{BB}} \\
\mathrm{EE}_{\mathrm{BS}}=\frac{\mathrm{SE}}{P_{C, \mathrm{BS}}}, \quad \mathrm{EE}_{\mathrm{UT}}=\frac{\mathrm{SE}}{P_{C, \mathrm{UT}}}
\end{gathered}
$$

where $N_{\mathrm{RF}}$ is the number of RF chains.

\section{Channel model}

The propagation of mmWave signals through an outdoor environment is accompanied by tiny reflection coefficients and sever path loss, and this leads to limited scattering clusters and spatially sparse channels. Thus, we adopt the widely used geometry-based channel model [32] for system performance evaluation. In this model, the MIMO channel matrix can be expressed as

$$
\mathbf{H}=\sqrt{\frac{N_{t} N_{r}}{P_{l} C}} \sum_{i=1}^{C} g_{i} \mathbf{v}_{r}\left(\theta_{i}\right) \mathbf{v}_{t}\left(\phi_{i}\right)^{H}
$$

where $\mathbf{H} \in \mathbb{C}^{N_{r} \times N_{t}}, C$ is the number of scattering clusters, $P_{l}$ is the path-loss, the $i^{\text {th }}$ cluster has path gain $g_{i}$, azimuth 


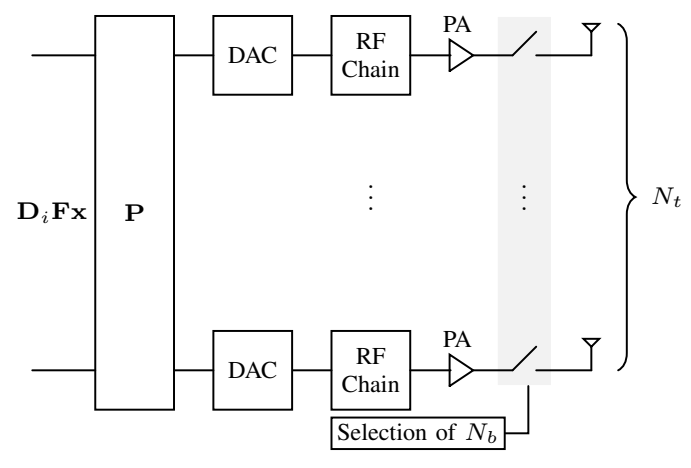

(a) BS circuitry

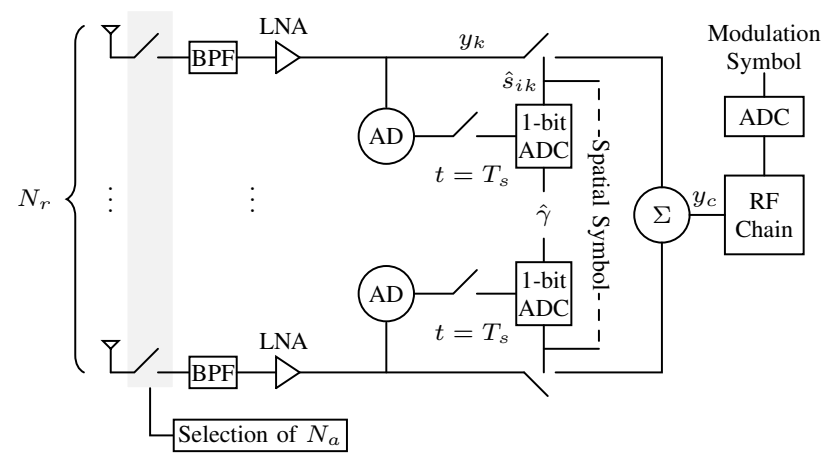

(b) UT circuitry of single RF (SRF) RSM

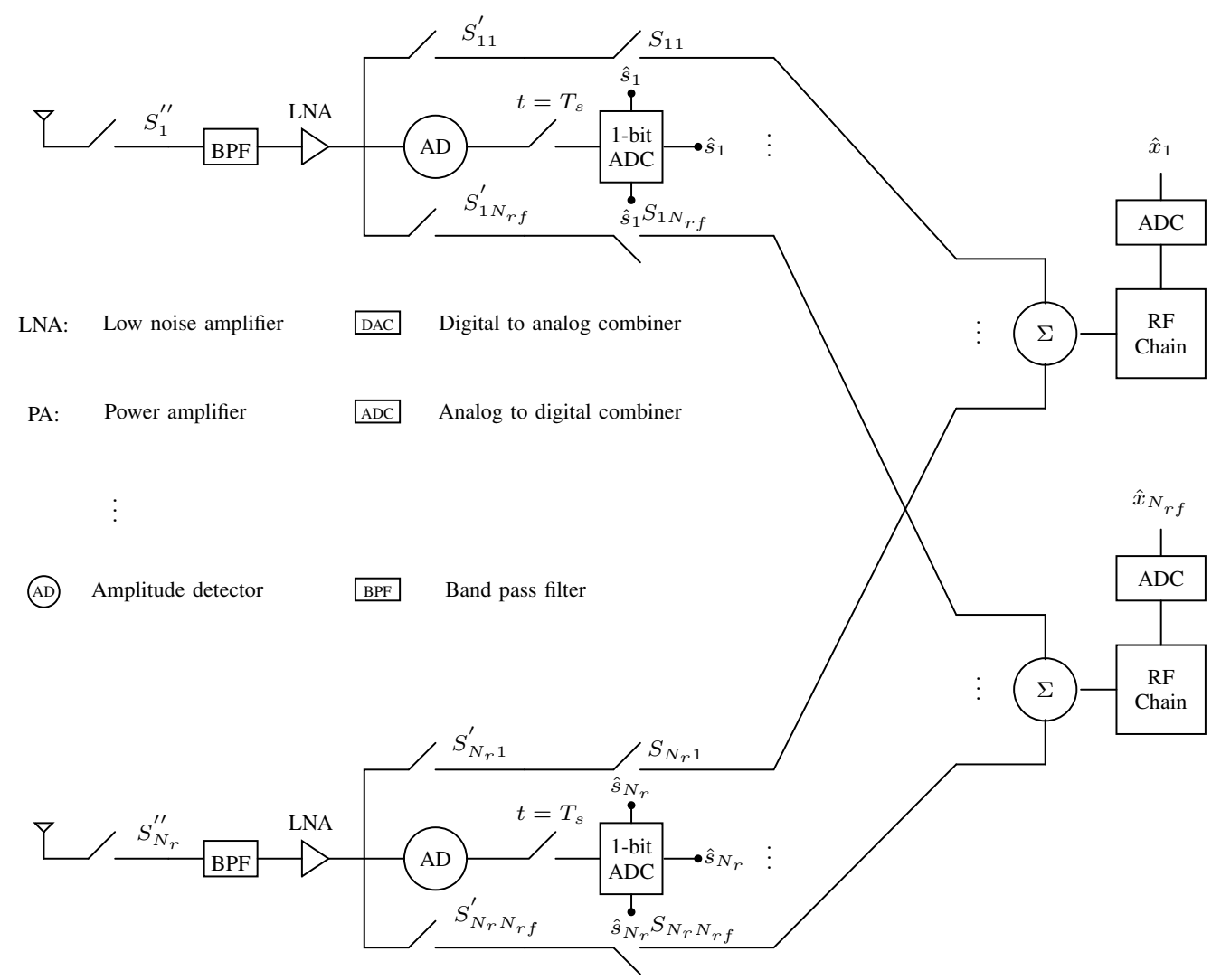

(c) UT circuitry of multiple RF (MRF) RSM

Fig. 1: Transceiver circuitries. The switch $S_{k}^{\prime \prime}$ controls the ON/OFF status of the $k^{\text {th }}$ antenna, $S_{i j}^{\prime}$ connects the $i^{\text {th }}$ received symbol to the $j^{\text {th }}$ RF chain. The switch status is determined according to an optimization algorithm. The switch $S_{i j}$ controls the combining method at the UT.

angles of departure and arrival $\phi_{i}$ and $\theta_{i}$, respectively and transmit and receive array response vectors $\mathbf{v}_{t}\left(\phi_{i}\right)$ and $\mathbf{v}_{r}\left(\theta_{i}\right)$, respectively. We consider uniform linear array (ULA) where the $\mathrm{N}$-elements response vector can be expressed as

$$
\mathbf{v}(\phi)=\frac{1}{\sqrt{N}}\left[1, e^{j k d \sin (\phi)}, \ldots, e^{j(N-1) k d \sin (\phi)}\right]^{T}
$$

where $k=\frac{2 \pi}{\lambda}$ and $d$ is the inter-elements spacing. ${ }^{1}$

\footnotetext{
${ }^{1}$ The proposed modulation scheme can be adapted to the wideband transmission by transmitting long symbols with many subcarriers (orthogonal frequency division multiplexing (OFDM)) or short symbols with single carrier and channel pre-equalization.
}

\section{E. System assumptions}

For the sake of reducing the training overhead, we exploit the channel reciprocity of mmWave propagation by applying time-division-duplex (TDD) protocol where the CSI is needed only at the BS. The BS can acquire the CSI during the uplink phase through low training overhead [8]. The BS transmits DL control signals to enable the detection parameters at the UTs as explained in [21].

\section{SINGLE USER}

\section{A. Single RF RSM with dependent combining (SRF-RSM-DC)}

In [21], the authors proposed SRF-RSM-DC scheme aims at improving the UT EE while attaining high SE where the 
incoming bit stream is modulated into two symbols in which $N_{a}$ bits are used to control the power level received by $N_{a}$ antennas (spatial symbol $\mathbf{s}_{i} \in \mathbb{R}^{N_{a} \times 1}$, can be detected by energy efficient devices) and the other bits are modulated based on $M$-ary constellation (modulation symbol $x_{j}$ ). The spatial symbol $\mathbf{s}_{i}$ comprises $N_{a}$ bits from the incoming binary bit stream where the $k^{\text {th }}$ spatial bit can be zero or one $\left(s_{i k} \in\{0,1\}\right)$. In the SRF-RSM-DC scheme, the transmit vector at the precoder input can be expressed as

$$
\mathbf{x}_{i}^{j}=\left\{\begin{array}{lll}
\mathbf{s}_{i} x_{j} & \text { if } & \mathbf{s}_{i} \neq \mathbf{0}_{N_{r}} \\
\mathbf{s}_{i} & \text { if } & \mathbf{s}_{i}=\mathbf{0}_{N_{r}}
\end{array}\right.
$$

where $x_{j}$ can not be transmitted with all zeros $\mathbf{s}_{i}$ to not loose the modulation symbol. In order to control the received signal power per antenna, we apply ZF precoder at the BS where the received signal vector can be expressed as

$$
\mathbf{y}=\mathbf{H}_{a} \mathbf{P x}_{i}^{j}+\mathbf{n}
$$

where $\mathbf{H}_{a} \in \mathbb{C}^{N_{a} \times N_{b}}$ is the channel matrix after selecting $N_{a}$ antennas at the UT and $N_{b}$ antennas at the BS, the design of $\mathbf{H}_{a}$ is illustrated in section $\mathrm{xx}, \mathbf{n} \in \mathbb{C}^{N_{a} \times 1}$ is independent and identically distributed noise vector with $\mathcal{C N}\left(0, \sigma^{2}\right)$ entries and $\mathbf{P} \in \mathbb{C}^{N_{b} \times N_{a}}$ is the $\mathrm{ZF}$ precoder that fixed during the coherence interval and satisfies

$$
\mathrm{E}\left[\left\|\mathbf{P} \mathbf{x}_{i}^{j}\right\|_{2}^{2}\right]=\operatorname{Tr}\left\{\mathbf{P} \mathbf{R}_{s s} \mathbf{P}^{H}\right\}=P_{t},
$$

where

$$
\mathbf{R}_{s s}=\mathrm{E}\left[\mathbf{s}_{i} \mathbf{s}_{i}^{H}\right]=\frac{1}{2^{N_{a}}} \sum_{i=1}^{2^{N_{a}}} \mathbf{s}_{i} \mathbf{s}_{i}^{H},
$$

The ZF precoding matrix can be expressed as

$$
\mathbf{P}=\sqrt{\alpha_{r} P_{t}} \mathbf{H}_{a}^{H}\left(\mathbf{H}_{a} \mathbf{H}_{a}^{H}\right)^{-1},
$$

where $\alpha_{r}$ is a normalization factor to adjust the transmit power. By substituting equation (13) in equation (11), we can express the factor $\alpha_{r}$ as

$$
\alpha_{r}=\frac{1}{\operatorname{Tr}\left\{\left(\mathbf{H}_{a} \mathbf{H}_{a}^{H}\right)^{-1} \mathbf{R}_{s s}\right\}}
$$

The signal received by the $k^{\text {th }}$ ARA can be expressed as

$$
y_{k}=\sqrt{\alpha_{r} P_{t}} s_{i k} x_{j}+n_{k}
$$

where equation (15) can be re-expressed as

$$
y_{k}= \begin{cases}\sqrt{\alpha_{r} P_{t}} x_{j}+n_{k} & \text { if } s_{i k}=1 \\ n_{k} & \text { if } s_{i k}=0\end{cases}
$$

where the spatial symbol controls the received power per ARA.

According to the energy efficient UT circuitry in Fig. 1b, we consider a reduced complexity detector where we can detect the spatial symbol using energy efficient analog devices and detect the modulation symbol using one RF chain only. In the spatial symbol detection, the AD connected to the $k^{\text {th }}$ ARA measures the amplitude of the received signal by this antenna that can be expressed as

$$
a_{k}= \begin{cases}\left|\sqrt{\alpha_{r} P_{t}} x_{j}+n_{k}\right| & \text { if } s_{i k}=1 \\ \left|n_{k}\right| & \text { if } s_{i k}=0\end{cases}
$$

and then, we compare the measured amplitude with a threshold to detect the $k^{\text {th }}$ spatial bit as follows

$$
\hat{s}_{i k}=\left\{\begin{array}{ll}
1 & \text { if } a_{k} \geqslant \gamma \\
0 & \text { if } a_{k}<\gamma
\end{array} .\right.
$$

In [21], a closed form expressions for the spatial symbol detection threshold $\gamma$ have been proved at different SNR regimes. The spatial error occurs when we transmit $s_{i k}=1$ and we detect $\hat{s}_{i k}=0$ is $P_{1}$ or vice versa is $P_{0}$. The average spatial bit error probability $\left(P_{s}\right)$ can be expressed as

$$
\begin{aligned}
& P_{s}=\frac{1}{2}\left(P_{1}+P_{0}\right) \\
& P_{1}=\operatorname{Pr}\left(a_{k}<\gamma \mid s_{i k}=1\right)=1-\mathcal{Q}_{1}\left(\frac{1}{\sigma} \sqrt{2} a_{h}, \frac{1}{\sigma} \sqrt{2} \gamma\right) \\
& P_{0}=\operatorname{Pr}\left(a_{k}>\gamma \mid s_{i k}=0\right)=\mathcal{Q}_{1}\left(\frac{1}{\sigma} \sqrt{2} a_{l}, \frac{1}{\sigma} \sqrt{2} \gamma\right)
\end{aligned}
$$

where $a_{k}$ has Ricean distribution [33] and $\mathcal{Q}_{1}($.$) denotes the$ first order Marcum Q-function and

$$
a_{h}=\sqrt{\alpha_{r} P_{t}}, \quad a_{l}=0, \quad \gamma=\frac{a_{h}+a_{l}}{2} .
$$

We exploit each ARA to enable detection of one spatial bit per transmission with success probabilities $\left(1-P_{1}\right)$ and $\left(1-P_{0}\right)$ and thus, the achievable spatial mutual information can be characterized by the binary asymmetric channel [34] mutual information expression that can be expressed as

$$
I_{S}^{\text {SRF-RSM-DC }}=N_{a}\left(\mathcal{H}\left(\frac{P_{0}+1-P_{1}}{2}\right)-\frac{\mathcal{H}\left(P_{0}\right)+\mathcal{H}\left(1-P_{1}\right)}{2}\right)
$$

where $\mathcal{H}(P)=-P \log _{2} P-(1-P) \log _{2}(1-P)$ is the the entropy function.

In the modulation symbol detection of the SRF-RSM-DC scheme, for the sake of not accumulating noise at the RF chain, the received signal per the ARA passes through the RF chain only if the detected spatial bit is one. This can be ensured through switches controlled by the detected spatial bits as illustrated in Fig. 1b. Let us consider the transmitted spatial symbol is $\mathbf{s}_{i}$ and the detected one is $\hat{\mathbf{s}}_{l}$ and thus, the combined signal at the RF chain can be expressed as

$$
y_{c}^{\text {SRF-RSM-DC }}=\hat{\mathbf{s}}_{l}^{T} \mathbf{y}=\sqrt{\alpha_{r} P_{t}} \hat{\mathbf{s}}_{l}^{T} \mathbf{s}_{i} x_{j}+\hat{\mathbf{s}}_{l}^{T} \mathbf{n}
$$

and the combined SNR can be expressed as

$$
\mathrm{SNR}_{i, l, c}^{\text {SRF-RSM-DC }}=\frac{\left(\hat{\mathbf{s}}_{l}^{T} \mathbf{s}_{i}\right)^{2}}{\max \left(\sum_{k=1}^{N_{a}} \hat{s}_{l k}, 1\right)} \frac{\alpha_{r} P_{t}}{\sigma^{2}} .
$$

The errors in the spatial symbol detection affects the combined SNR and thus, the modulation symbol bit error probability can be expressed as

$$
\begin{gathered}
P_{m}^{\text {SRF-RSM-DC }}=\sum_{i=1}^{2^{N_{a}}-1} \operatorname{Pr}\left(\mathbf{s}_{i}\right) \sum_{l=1}^{2^{N_{a}}}\left(\operatorname{Pr}\left(\hat{\mathbf{s}}_{l} \mid \mathbf{s}_{i}\right)\right. \\
\left.\times \operatorname{BEP}\left(x_{j} \in \mathcal{C}_{M} \mid \mathrm{SNR}_{i, l, c}^{\text {SRF-RSM-DC }}\right)\right) \\
\operatorname{Pr}\left(\hat{\mathbf{s}}_{l} \mid \mathbf{s}_{i}\right)=\prod_{k=1}^{N_{a}} \operatorname{Pr}\left(a_{k} \underset{\hat{s}_{l k}=0}{\gtrless} \gamma \mid s_{i k}\right), \operatorname{Pr}\left(\mathbf{s}_{i}\right)=\frac{1}{2^{N_{a}}-1}
\end{gathered}
$$


where $\operatorname{BEP}\left(x_{j} \in \mathcal{C}_{M}\right)$ is the bit error probability of $M$-ary modulation scheme. Since the received power level per ARA affects the quality of the spatial symbol detection, constant amplitude modulation schemes ( $M-\mathrm{PSK}$ ) are shown in [21] to achieve the best performance as the received power does not depend on the constellation point. Since we exploit the diversity of the multiple UT antennas to detect single modulation symbol only, the modulation symbol mutual information can be given by the multiple-input-single-output expression taking into account the shaping loss resulting from transmitting $M$-PSK symbols as

$$
\begin{aligned}
I_{M}^{\text {SRF-RSM-DC }} & =\left(1-2^{-N_{a}}\right) \sum_{i=1}^{2^{N_{a}}-1} \operatorname{Pr}\left(\mathbf{s}_{i}\right) \sum_{l=1}^{2^{N_{a}}} \operatorname{Pr}\left(\hat{\mathbf{s}}_{l} \mid \mathbf{s}_{i}\right) \\
& \times I_{M-\mathrm{PSK}}\left(\mathrm{SNR}_{i, l, c}^{\text {SRF-RSM-DC }}\right) .
\end{aligned}
$$

where $I_{M-\mathrm{PSK}}$ at given SNR can be expressed as [35]

$$
\begin{aligned}
& I_{M-\mathrm{PSK}}(\mathrm{SNR})=\beta^{2} \log _{2} e \\
& -\int_{0}^{\infty} r e^{-\frac{r^{2}+\beta^{2}}{2}} \mathrm{I}_{0}(\beta r) \log _{2} \mathrm{I}_{0}(\beta r) d r
\end{aligned}
$$

where $\beta^{2}=2 \mathrm{SNR}$ and the SE of the SRF-RSM-DC scheme can be expressed as

$$
\mathrm{SE}^{\mathrm{SRF}-\mathrm{RSM}-\mathrm{DC}}=I_{S}^{\mathrm{SRF}-\mathrm{RSM}-\mathrm{DC}}+I_{M}^{\text {SRF-RSM-DC }} .
$$

\section{B. Single RF RSM with independent combining (SRF-RSM- IC)}

In the SRF-RSM-DC scheme, we cannot transmit $x_{j}$ with all zeros $\mathbf{s}_{i}$. The probability of getting $\mathbf{s}_{i}=\mathbf{0}_{N_{a}}$ is $1 / 2^{N_{a}}$ and thus, at small $N_{a}$, we do not transmit $x_{j}$ with high probability and this could degrade the SE. Therefore, we propose a SRFRSM-IC scheme where we detect $x_{j}$ and $\mathbf{s}_{i}$ independently. In this scheme, $s_{i k} \in\left\{0<\alpha_{0}<0.5,1-\alpha_{0}\right\}$ and the received modulation symbol per ARA always passes through the RF chain regardless the value of $\hat{s}_{l k}$. This can be achieved by the flexible circuitry in Fig. 1b through keeping the switches between $y_{k}$ and the RF chain always closed. Accordingly, we can transmit $x_{j}$ with all zeros spatial bits and the transmit signal vector at the precoder input can be expressed as

$$
\mathbf{x}_{i}^{j}=\mathbf{s}_{i} x_{j} \quad \forall \mathbf{s}_{i} .
$$

Since we have the same UT hardware constraints as the SRF-RSM-DC scheme, we apply the spatial symbol detector as in equation (18) that leads to same expressions of spatial errors and spatial mutual information $\left(I_{S}^{\text {SRF-RSM-IC }}\right)$ as depicted in equations (19) and (21), respectively. In this case, the received amplitudes depend on $\alpha_{0}$ as follows

$$
a_{h}=\left(1-\alpha_{0}\right) \sqrt{\alpha_{r} P_{t}}, \quad a_{l}=\alpha_{0} \sqrt{\alpha_{r} P_{t}}
$$

In the SRF-RSM-IC scheme, the combined signal does not depend on the detected spatial symbol and all the received signals per ARAs are combined together as follows

$$
y_{c}^{\text {SRF-RSM-IC }}=\mathbf{1}_{N_{a}}^{T} \mathbf{y}=\sqrt{\alpha_{r} P_{t}} \mathbf{1}_{N_{a}}^{T} \mathbf{s}_{i} x_{j}+\mathbf{1}_{N_{a}}^{T} \mathbf{n}
$$

and the combined SNR can be expressed as

$$
\mathrm{SNR}_{i, c}^{\mathrm{SRF}-\mathrm{RSM}-\mathrm{IC}}=\frac{\left(\mathbf{1}_{N_{a}}^{T} \mathbf{s}_{i}\right)^{2}}{N_{a}} \frac{\alpha_{r} P_{t}}{\sigma^{2}} .
$$

As the combined SNR is free from spatial errors, the modulation symbol bit error probability and the modulation mutual information in equations (24) and (25), respectively, can be re-expressed as

$$
\begin{aligned}
& P_{m}^{\text {SRF-RSM-IC }}=\sum_{i=1}^{2^{N_{a}}} \operatorname{Pr}\left(\mathbf{s}_{i}\right) \\
& \times \operatorname{BEP}\left(x_{j} \in M-\mathrm{PSK} \mid \mathrm{SNR}_{i, c}^{\mathrm{SRF}-\mathrm{RSM}-\mathrm{IC}}\right) \text {, } \\
& I_{M}^{\mathrm{SRF}-\mathrm{RSM}-\mathrm{IC}}=\sum_{i=1}^{2^{N_{a}}} \operatorname{Pr}\left(\mathbf{s}_{i}\right) I_{M-\mathrm{PSK}}\left(\mathrm{SNR}_{i, c}^{\mathrm{SRF}-\mathrm{RSM}-\mathrm{IC}}\right) .
\end{aligned}
$$

We design the spatial symbol power level factor $\alpha_{0}$ to maximize the $\mathrm{SE}$ as follows

$$
\begin{array}{ll}
\underset{\alpha_{0}}{\operatorname{maximize}} & I_{S}^{\mathrm{SRF}-\mathrm{RSM}-\mathrm{IC}}+I_{M}^{\mathrm{SRF}-\mathrm{RSM}-\mathrm{IC}} \\
\text { subject to } & 0 \leq \alpha_{0} \leq .5 .
\end{array}
$$

where the optimal value of $\alpha_{0}$ in equation (33) can be obtained using the bisection method [36]. The SE of the SRF-RSM-IC scheme can be expressed as

$$
\mathrm{SE}^{\mathrm{SRF}-\mathrm{RSM}-\mathrm{IC}}=I_{S}^{\mathrm{SRF}-\mathrm{RSM}-\mathrm{IC}}+I_{M}^{\mathrm{SRF}-\mathrm{RSM}-\mathrm{IC}} .
$$

\section{Single RF Conventional modulation (SRF-CM)}

The badly conditioned and highly spatially sparse mmWave channels may lead to activating one ARA only and thus, transmitting one modulation symbol without spatial modulated bits (CM) could achieve better SE than RSM. The flexible architecture in Fig. $1 \mathrm{~b}$ can be adapted to $\mathrm{CM}$ by activating one receive antenna and transmit fixed spatial bit equals to one. Assuming ZF precoding, the received signal per the active antenna and the $\mathrm{SNR}_{c}$ can be expressed as

$$
\begin{aligned}
& y_{c}=\sqrt{\alpha P_{t}} x_{j}+n, \quad \operatorname{SNR}_{c}=\frac{\alpha_{c} P_{t}}{\sigma^{2}}, \\
& \alpha_{c}=\frac{1}{\operatorname{Tr}\left\{\left(\mathbf{H}_{a} \mathbf{H}_{a}^{H}\right)^{-1}\right\}} .
\end{aligned}
$$

In $\mathrm{CM}$, we do not transmit spatial symbol and thus, the constant amplitude constellation constraint is not necessary and $M$-quadrature-amplitude-modulation ( $M-\mathrm{QAM})$ modulation scheme achieves the highest $\mathrm{SE}$ in this case. The bit error probability and the SE of the CM scheme assuming the shaping loss of $M-$ QAM [37] symbols can be expressed as

$$
\begin{gathered}
P_{m}^{\mathrm{SRF}-\mathrm{CM}}=\mathrm{BEP}\left(x_{j} \in M-\mathrm{QAM} \mid \mathrm{SNR}_{c}\right), \\
\mathrm{SE}^{\mathrm{SRF}-\mathrm{CM}}=\log _{2}\left(1+\frac{\alpha_{c} P_{t}}{\sigma^{2}} / \frac{\pi e}{6}\right) .
\end{gathered}
$$

\section{Multiple RF RSM with independent combining (MRF- RSM-IC)}

We can transmit multiple modulation symbols and single spatial symbol per channel use by employing multiple RF chains at the UT. Therefore, the SE can be affected more by transmitting the modulation symbols and this motivates the IC scheme where the modulation symbols are always transmitted 
regardless the spatial symbol value. In this case, the transmit signal vector at the precoder input can be expressed as

$$
\mathbf{x}_{i}^{j}=\mathbf{\Lambda}\left(\mathbf{s}_{i}\right) \mathbf{F} \mathbf{x}_{j}
$$

where $\boldsymbol{\Lambda}\left(\mathbf{s}_{i}\right) \in \mathbb{R}^{N_{a} \times N_{a}}$ diagonal matrix whose entries $\boldsymbol{\Lambda}_{i i} \in\left\{\alpha_{0}, 1-\alpha_{0}\right\}, \mathbf{x}_{j} \in \mathbb{C}^{N_{m} \times 1}, N_{m} \leqslant N_{r f}$ is the number of the transmitted modulation symbols and $\mathbf{F} \in \mathbb{R}^{N_{a} \times N_{m}}$ is orthogonal mapping matrix that can be expressed as

$$
\mathbf{F}_{n m}= \begin{cases}1 & \text { if } n-m=l N_{m} \\ & l=0, \cdots,\left\lfloor\frac{N_{a}-1}{N_{m}}\right\rfloor \\ 0 \quad & \text { else }\end{cases}
$$

The transmit covariance matrix $\mathbf{R}_{s s}$ can be expressed as

$\mathbf{R}_{s s}=\mathrm{E}\left[\boldsymbol{\Lambda}\left(\mathbf{s}_{i}\right) \mathbf{F F}{ }^{H} \boldsymbol{\Lambda}\left(\mathbf{s}_{i}\right)^{H}\right]=\frac{1}{2^{N_{a}}} \sum_{i=1}^{2^{N_{a}}} \boldsymbol{\Lambda}\left(\mathbf{s}_{i}\right) \mathbf{F} \mathbf{F}^{H} \boldsymbol{\Lambda}\left(\mathbf{s}_{i}\right)^{H}$

The detection of the spatial symbol and the spatial mutual information $\left(I_{S}^{\mathrm{MRF}-\mathrm{RSM}-\mathrm{IC}}\right)$ are the same as single RF case. The combined signal vector $\mathbf{y}_{c}$ of the modulation symbols and the combined $\mathrm{SNR}_{c, l}$ of the $l^{\text {th }}$ modulation symbol can be expressed as

$$
\begin{aligned}
& \mathbf{y}_{c}=\mathbf{F}^{T}\left(\sqrt{\alpha_{r} P_{t}} \boldsymbol{\Lambda}\left(\mathbf{s}_{i}\right) \mathbf{F} \mathbf{x}_{j}+\mathbf{n}\right) \\
& \mathrm{SNR}_{c, l}=\frac{\left(\left[\mathbf{F}^{T} \boldsymbol{\Lambda}\left(\mathbf{s}_{i}\right) \mathbf{F}\right]_{l l}\right)^{2}}{\left[\mathbf{F}^{T} \mathbf{F}\right]_{l l}} \frac{\alpha_{r} P_{t}}{\sigma^{2}}
\end{aligned}
$$

The modulation symbols mutual information and the SE of the MRF-RSM-IC scheme can be expressed as

$$
\begin{aligned}
I_{M}^{\mathrm{MRF}-\mathrm{RSM}-\mathrm{IC}} & =\sum_{l=1}^{N_{m}} \sum_{i=1}^{2^{N_{a}}} \operatorname{Pr}\left(\mathbf{s}_{i}\right) I_{M-\mathrm{PSK}}\left(\mathrm{SNR}_{c, l}\right), \\
\mathrm{SE}^{\mathrm{MRF}-\mathrm{RSM}-\mathrm{IC}} & =I_{S}^{\mathrm{MRF}-\mathrm{RSM}-\mathrm{IC}}+I_{M}^{\mathrm{MRF}-\mathrm{RSM}-\mathrm{IC}} .
\end{aligned}
$$

\section{E. Multiple RF Conventional modulation (MRF-CM)}

In CM scheme with multiple RF at the UT, we perform antenna selection as in Algorithm 1 and transmit $N_{m} \leqslant N_{r f}$ $M$-QAM symbols to maximize the SE that can be expressed as

$$
\mathrm{SE}^{\mathrm{MRF}-\mathrm{CM}}=\sum_{l=1}^{N_{m}} \log _{2}\left(1+\frac{\alpha_{c} P_{t}}{\sigma^{2}} / \frac{\pi e}{6}\right) .
$$

The outdoor propagation at mmWave frequencies is spatially sparse and thus, the channel is badly conditioned and tends to be rank deficient when activating all of the receive antennas. However, we can ensure full-rank channel by selecting the receive antennas set that maximizes the SE and thus, we can apply the ZF precoder. On the other hand, FD BS is power consuming at mmWave band and hence, TAS can be applied to improve the $\mathrm{EE}$ at the $\mathrm{BS}\left(\mathrm{EE}_{\mathrm{BS}}\right)$ such that not all of the RF chains at the BS are active simultaneously. For the sake of reducing the computational complexity, we start with the RAS assuming all of the transmit antennas are active and after that, we apply the TAS algorithm. The number of search

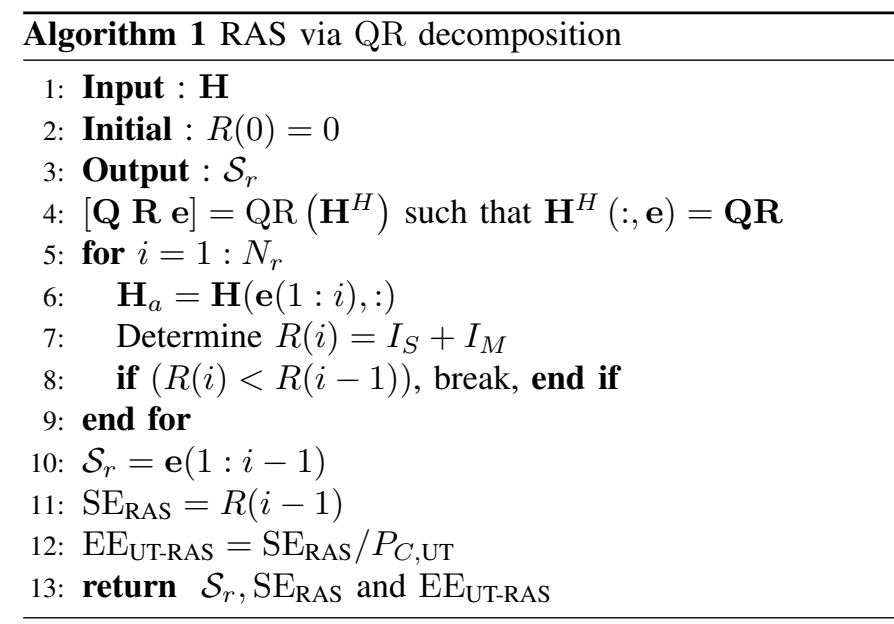

iterations needed for the proposed RAS+TAS algorithms $\left(n_{p}\right)$ and for exhaustive search $\left(n_{e}\right)$ can be expressed as

$$
\begin{aligned}
& n_{p}=N_{r}+N_{t} \\
& n_{e}=\sum_{i=1}^{N_{r}}\left(\begin{array}{c}
N_{r} \\
i
\end{array}\right) \times \sum_{j=i}^{N_{t}}\left(\begin{array}{c}
N_{t} \\
j
\end{array}\right) .
\end{aligned}
$$

As an illustrative example to the significant reduction in the search complexity, let use consider realistic antennas numbers $\left(N_{t}=128\right.$ and $\left.N_{r}=16\right)$ and hence, $n_{p}=144$ and $n_{e} \approx 2.2 \times$ $10^{43}$. The RAS and TAS algorithms are determined at the BS and then, the UT be informed about the ARA through a control channel. In the sequel, we present fast and efficient antenna selection algorithms and we compare their performance and complexity with the exhaustive search based selection.

\section{F. Receive antenna selection}

In Algorithm 1, we apply the QR decomposition [38] on the channel matrix such that the power received per antenna $\left(P_{r}\right)$ can be expressed in terms of the upper triangular matrix $\mathbf{R}$ (resulting from the $\mathrm{QR}$ decomposition) as follows

$$
\begin{aligned}
P_{r} \propto \frac{1}{\operatorname{Tr}\left\{\left(\mathbf{H H}^{H}\right)^{-1}\right\}} & =\frac{1}{\operatorname{Tr}\left\{\left(\mathbf{R}^{H} \mathbf{R}\right)^{-1}\right\}} \\
& \propto \prod_{i=1}^{N_{r}} \mathbf{R}_{i i}^{2} .
\end{aligned}
$$

where $\mathbf{R}_{i i}$ is the $i^{\text {th }}$ diagonal entry of $\mathbf{R}$. The diagonal entries of $\mathbf{R}$ are sorted in descending order and each entry $\mathbf{R}_{i i}$ corresponds to specific receive antenna with index $\mathbf{e}(i)$. Thus, we keep adding the receive antennas with large $\mathbf{R}_{i i}$ to the set $\mathcal{S}_{r}$ and evaluate the SE at each iteration. Finally, the algorithm stops when the SE degrades and thus, set $\mathcal{S}_{r}$ comprises the ARA.

\section{G. Transmit antenna selection}

As shown in Algorithm 2, we apply the QR decomposition on the matrix $\mathbf{H}\left(\mathcal{S}_{t},:\right)$ to sort the transmit antennas in a similar way as in equation (46). Initially, we assume all of the transmit antennas are active and then, we deactivate one antenna per 


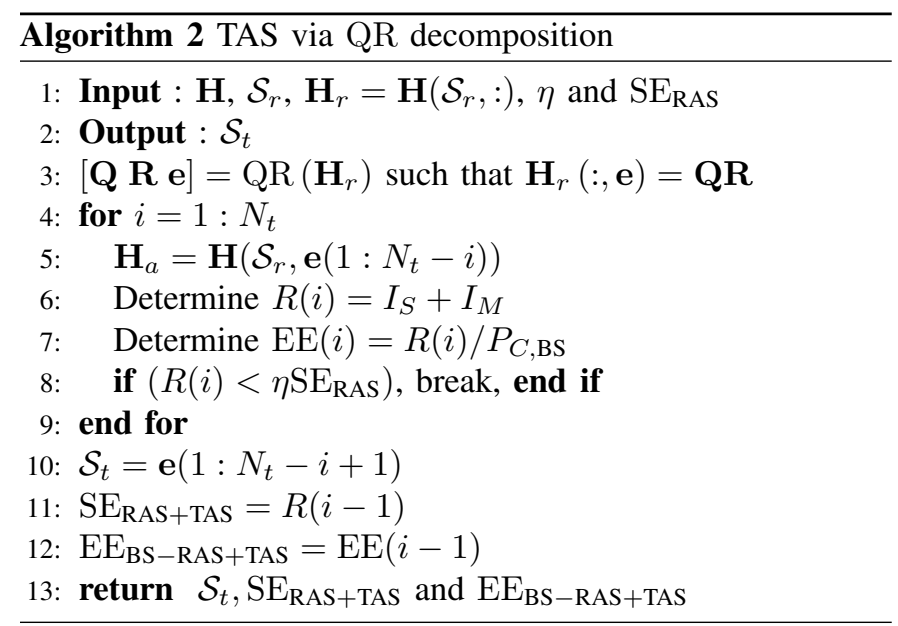

iteration that corresponds to minimum $\mathbf{R}_{i i}$ value. Deactivating transmit antennas reduces the $\mathrm{SE}$ and improves the $\mathrm{EE}_{\mathrm{BS}}$ and thus, we keep deactivating the transmit antennas such that the $\mathrm{SE}$ is greater than or equals to threshold $\left(\eta \mathrm{SE}_{\mathrm{RAS}}\right)$ where $(0 \leq \eta \leq 1)$. Finally, the set $\mathcal{S}_{t}$ includes the active transmit antennas indices.

\section{Multi-USER RSM}

In this section, we consider single BS serves $N_{U}$ users each has $N_{r}$ antennas in the DL by applying ZF precoding. We provide novel joint transmit power allocation and UTs antenna selection algorithm to maximize the weighted sum SE assuming RSM transmission scheme. At a given number of users and set of antennas per user, we formulate the power allocation as non convex optimization problem. Next, we propose an alternating optimization algorithm to convert the non convex problem into series of convex problems. The alternating optimization is an iterative algorithm and at each iteration we solve convex optimization problem. Finally, we compare the performance and the convergence of the proposed alternating optimization with the successive convex approximation framework.

\section{A. Joint MU power allocation and antenna selection}

For simplicity, let us consider the system model assuming single RF chain at each UT; however, the analysis can be straightforward extended to multiple RF chains. The BS broadcasts different SM symbol to each user such that the transmit signal after precoding can be expressed as

$$
\begin{aligned}
& \mathbf{x}_{t}=\sum_{k=1}^{N_{u}} \sqrt{b_{k}} \mathbf{P}_{k} \mathbf{s}_{i, k} x_{j, k}, \quad \text { with } \\
& \mathrm{E}\left[\mathbf{x}_{t}^{H} \mathbf{x}_{t}\right]=\sum_{k=1}^{N_{U}} b_{k} \operatorname{Tr}\left\{\mathbf{P}_{k} \mathbf{R}_{s_{k}, s_{k}} \mathbf{P}_{k}^{H}\right\}=\sum_{k=1}^{N_{U}} b_{k} \mu_{k}=P_{t}, \\
& \mathbf{R}_{s_{k}, s_{k}}=\frac{1}{2^{N_{a, k}}} \sum_{i=1}^{2^{N_{a, k}}} \mathbf{s}_{i, k}, \mathbf{s}_{i, k}^{H}, \quad \mathbf{P}=\mathbf{H}_{a}^{H}\left(\mathbf{H}_{a} \mathbf{H}_{a}^{H}\right)^{-1}, \\
& \mathbf{P}_{k}=\mathbf{P}\left(:, 1+\sum_{i=0}^{k-1} N_{a, i}: \sum_{i=1}^{k} N_{a, i}\right), \quad N_{a, 0}=0 .
\end{aligned}
$$

where, per the $k^{\text {th }}$ user, $x_{j, k}$ is $M$-PSK modulation symbol, $\mathbf{s}_{i, k} \in \mathbb{R}^{N_{a, k} \times 1}$ is the spatial symbol and its entries $s_{i, k}(n) \in\left\{0<\alpha_{0, k}<0.5,1-\alpha_{0, k}\right\}, \forall n=$ $1, \cdots, N_{a, k}$ (number of active antennas at the UT), $\mathbf{P}_{k} \in$ $\mathbb{C}^{N_{t} \times N_{a, k}}$ is the sub-precoding matrix, $b_{k}$ is power allocation factor, $\mathbf{R}_{s_{k}, s_{k}}$ represents the spatial symbol covariance matrix, $\mathbf{P} \in \mathbb{C}^{N_{t} \times \sum_{k=1}^{N_{u}} N_{a, k}}$ is the multi-user ZF precoding matrix, $\mathbf{H}_{a}=\left[\mathbf{H}_{a, 1}, \cdots, \mathbf{H}_{a, N_{U}},\right]^{T} \in \mathbb{C}^{\sum_{k=1}^{N_{u}} N_{a, k} \times N_{t}}$ is the channel matrix of all the users after applying the antenna selection and $\mathbf{H}_{a, k}$ represents the user channel matrix after selecting antennas from the matrix $\mathbf{H}_{k}$. The signal received by the $k^{\text {th }}$ user can be expressed as expressed as

$$
\begin{aligned}
\mathbf{y}_{k} & =\mathbf{H}_{a, k}\left(\sum_{k=1}^{N_{u}} \sqrt{b_{k}} \mathbf{P}_{k} \mathbf{s}_{i, k} x_{j, k}\right)+\mathbf{n}_{k} \\
\mathbf{y}_{k} & =\sqrt{b_{k}} \mathbf{s}_{i, k} x_{j, k}+\mathbf{n}_{k}
\end{aligned}
$$

where the combined SNR at the $k^{\text {th }}$ user can be expressed as

$$
\mathrm{SNR}_{i, k}=\frac{\left(\mathbf{1}_{N_{a, k}}^{T} \mathbf{s}_{i, k}\right)^{2}}{N_{a, k}} \frac{b_{k}}{\sigma^{2}} .
$$

The received signal in Eq. (48) is similar to that of the single user case in Eq. (15). Thus, the $k^{\text {th }}$ mutual information for the spatial symbol $\left(I_{S, k}\right)$ and for the modulation symbol $\left(I_{M, k}\right)$ can be expressed in a similar way as in Eq. (21) and Eq. (32), respectively as

$$
\begin{aligned}
I_{S, k} & =N_{a, k} \times \\
& \left(\mathcal{H}\left(\frac{P_{0, k}+1-P_{1, k}}{2}\right)-\frac{\mathcal{H}\left(P_{0, k}\right)+\mathcal{H}\left(1-P_{1, k}\right)}{2}\right), \\
I_{M, k} & =\sum_{i=1}^{2^{N_{a, k}} \operatorname{Pr}\left(\mathbf{s}_{i, k}\right) I_{M-\text { PSK }}\left(\mathrm{SNR}_{i, k}\right) .}
\end{aligned}
$$

where the error probabilities can be expressed as

$$
\begin{aligned}
P_{1, k} & =1-\mathcal{Q}_{1}\left(\frac{1}{\sigma} \sqrt{2} a_{h, k}, \frac{1}{\sigma} \sqrt{2} \gamma_{k}\right), \\
P_{0, k} & =\mathcal{Q}_{1}\left(\frac{1}{\sigma} \sqrt{2} a_{l . k}, \frac{1}{\sigma} \sqrt{2} \gamma_{k}\right), \\
a_{h, k} & =\left(1-\alpha_{0, k}\right) \sqrt{b_{k}}, \quad a_{l, k}=\alpha_{0, k} \sqrt{b_{k}} \text { and } \\
\gamma_{k} & =\frac{a_{h, k}+a_{l, k}}{2},
\end{aligned}
$$

and the $\mathrm{SE}$ of the $k^{\text {th }}$ user can be expressed as

$$
\mathrm{SE}_{k}=I_{S, k}+I_{M, k}
$$

In Algorithm 3, we jointly optimize number of users, set of active users antennas, high and low spatial amplitude levels and the power allocated for each user to maximize the weighted sum SE. At first, we apply the QR decomposition on $\mathbf{H}=\left[\mathbf{H}_{1}, \cdots, \mathbf{H}_{N_{U}}\right]^{T}$ to sort the UT antennas indices. The set $\mathcal{A}$, in line 4 of Algorithm 3, includes the sorted UTs antenna indices such that $\mathcal{A}(1)$ refers to the antenna index with maximum $\mathbf{R}_{i i}$ value. Next, we add one antenna per iteration from set $\mathcal{A}$. For the resulting user distribution and active antennas per user, we allocate the transmit power $P_{t, k}=b_{k} \mu_{k}$ and design the spatial levels $\alpha_{0, k}$ to maximize the 


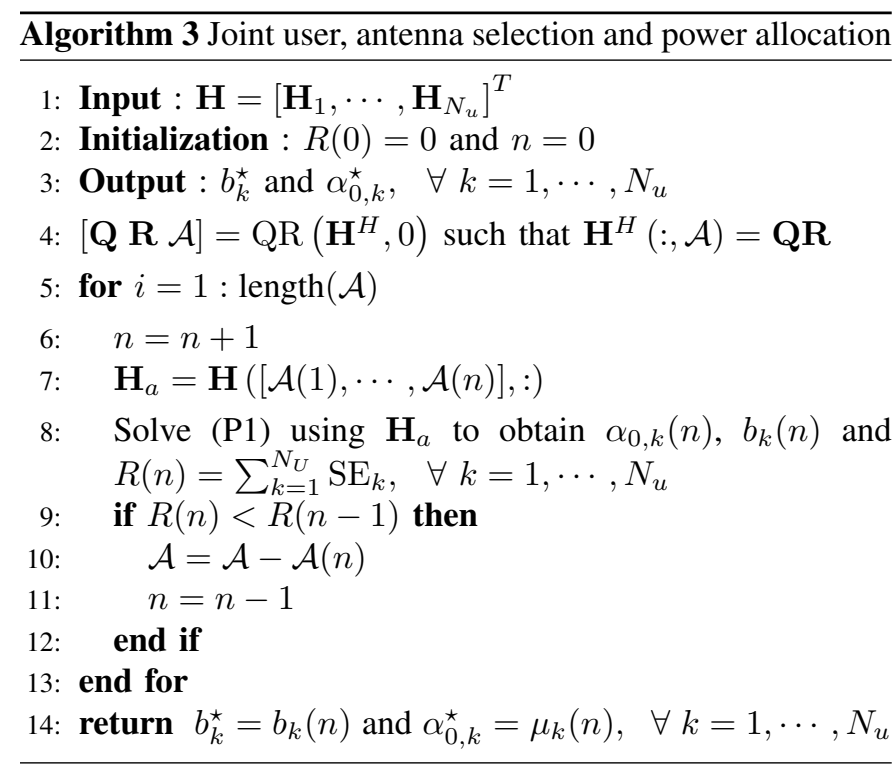

weighted sum SE. Hence, the power allocation optimization problem can be expressed as

$$
\text { (P1) }\left\{\begin{array}{cl}
\underset{b_{1}, \cdots, b_{N_{u}}, \alpha_{0,1}, \cdots, \alpha_{0, N_{u}}}{\operatorname{maximize}} & \sum_{k=1}^{N_{U}} \rho_{k}\left(I_{M, k}+I_{S, k}\right) \\
\text { subject to } & \sum_{k=1}^{N_{U}} b_{k} \mu_{k}=P_{t}, \\
& 0 \leqslant \alpha_{0, k} \leqslant 0.5, \\
& \forall k=1, \cdots, N_{U},
\end{array}\right.
$$

where $\rho_{k}$ is the SE weight per the $k^{\text {th }}$ user and $\mu_{k}$ can be expressed as

$$
\begin{aligned}
\mu_{k} & =\left(\frac{\alpha_{0, k}^{2}+\left(1-\alpha_{0, k}\right)^{2}}{2}-\frac{1}{4}\right) \operatorname{Tr}\left\{\mathbf{P}_{k}^{H} \mathbf{P}_{k}\right\} \\
& +\frac{1}{4} \operatorname{Tr}\left\{\mathbf{1}_{N_{a, k}} \mathbf{P}_{k}^{H} \mathbf{P}_{k}\right\} .
\end{aligned}
$$

At each iteration, we determine the weighted sum SE resulting from the solution of (P1) and if it degrades, we discard the antenna added at this iteration. We repeat the above procedure with all antennas in the set $\mathcal{A}$.

From Eq. (50), the spatial and modulation symbols mutual information are not convex functions in the power allocation variables $b_{k}$ and the spatial level factors $\alpha_{0, k}$ and thus, the objective function of problem (P1) is non-convex. Moreover, problem (P1) has non-convex joint power constraint. In the sequel, we propose alternating optimization algorithm to convert the non-convex problem (P1) into convex optimization problems at each iteration. After that, we compare the performance and the convergence of the proposed algorithm with successive convex approximation scheme.

\section{B. MU power allocation using alternating optimization}

In Algorithm 4, we propose an alternating optimization method to solve problem (P1). First, we consider arbitrary values of the spatial levels $\alpha_{0, k}$. Thus, the constraint of problem (P1) become linear in $b_{k}$. However, the objective function still not convex. Therefore, we propose piecewise
Algorithm 4 Power allocation using the proposed alternating optimization

1: Initialization: Consider any value of $\alpha_{0, k}$ such that $0 \leq$ $\alpha_{0, k} \leq 0.5 \forall k=1, \cdots, N_{U}$ and $i=0$

2: $i=i+1$

3: Use the values of $\alpha_{0, k}$ to solve problem (P1) and hence, determine the transmit power per user $P_{t, k}(i)$

4: Use the obtained values of $P_{t, k}(i)$ from step 3 to solve problem (P1) and hence, determine the updated $\alpha_{0, k}$ and $b_{k}$

5: Go to step 2 until $P_{t, k}(i)-P_{t, k}(i-1) \leq \epsilon$

approximations for the modulation and the spatial symbols mutual information as follows

$$
\begin{gathered}
\tilde{I}_{M, k}=\min _{a_{n}}\left\{I_{M,\left.k\right|_{b_{k}=a_{n}}}+\frac{d}{d b_{k}} I_{M,\left.k\right|_{b_{k}=a_{n}}}\left(b_{k}-a_{n}\right)\right\}, \\
a_{0}=0, a_{n+1}=a_{n}+\frac{K}{\left|\frac{d^{2}}{d b_{k}^{2}} I_{M,\left.k\right|_{b_{k}=a_{n}}}\right|}, n=0, \cdots, N .
\end{gathered}
$$

where $K$ is a constant and $N$ is the number of piecewise functions,

$$
\tilde{I}_{S, k}=N_{a, k} \min \left\{1, I_{S, 1 k}, I_{S, 2 k}\right\},
$$

where $I_{S, 1 k}$ and $I_{S, 2 k}$ are tangents to $I_{S, k}$ and passes through $I_{S, k}=0$ and $I_{S, k}=0.9$, respectively. At given spatial levels $\alpha_{0, k}$ and thanks to the approximations in Eq. (55) and Eq. (56), we formulate problem (P1) as convex program

$$
\text { (P2) } \begin{cases}\underset{b_{1}, \cdots, b_{N_{u}}}{\operatorname{maximize}} & \sum_{k=1}^{N_{U}} \rho_{k}\left(\tilde{I}_{M, k}+\tilde{I}_{S, k}\right) \\ \text { subject to } & \sum_{k=1}^{N_{U}} b_{k} \mu_{k}=P_{t},\end{cases}
$$

that can be efficiently solved to determine the transmit power allocated for the $k^{\text {th }}$ user $P_{t, k}=b_{k} \mu_{k}$ as illustrated in step 3 of Algorithm 4. After that, we solve problem (P1) using the obtained $P_{t, k}$ from step 3 to determine the updated values of $b_{k}$ and $\alpha_{0, k}$ as

$$
\text { (P3) }\left\{\begin{array}{cl}
\underset{b_{1}, \cdots, b_{N_{U}}, \alpha_{0,1}, \cdots, \alpha_{0, N_{u}}}{\operatorname{maximize}} & \sum_{k=1}^{N_{U}} \rho_{k}\left(I_{M, k}+I_{S, k}\right) \\
\text { subject to } & b_{k} \mu_{k}=P_{t, k}, \\
& 0 \leqslant \alpha_{0, k} \leqslant 0.5, \\
& \forall k=1, \cdots, N_{U} .
\end{array}\right.
$$

Problem (P3) has individual power constraints and the objective function is sum of independent mutual information per user. Thus, we formulate problem (P3) as $N_{U}$ sub-problems each can be expressed as

$$
\text { (P4) }\left\{\begin{array}{ll}
\underset{b_{k}, \alpha_{0}, k}{\operatorname{maximize}} & I_{M, k}+I_{S, k} \\
\text { subject to } & b_{k}=\frac{P_{t, k}}{\mu_{k}}, \\
& 0 \leqslant \alpha_{0, k} \leqslant 0.5,
\end{array}, k=1, \cdots, N_{U}\right.
$$

where the optimal value of $\alpha_{0, k}$ can be obtained using the bisection methods [36] and $b_{k}=P_{t, k} / \mu_{k}$. Finally, we repeat steps 3 and 4 of Algorithm 4 until convergence. 


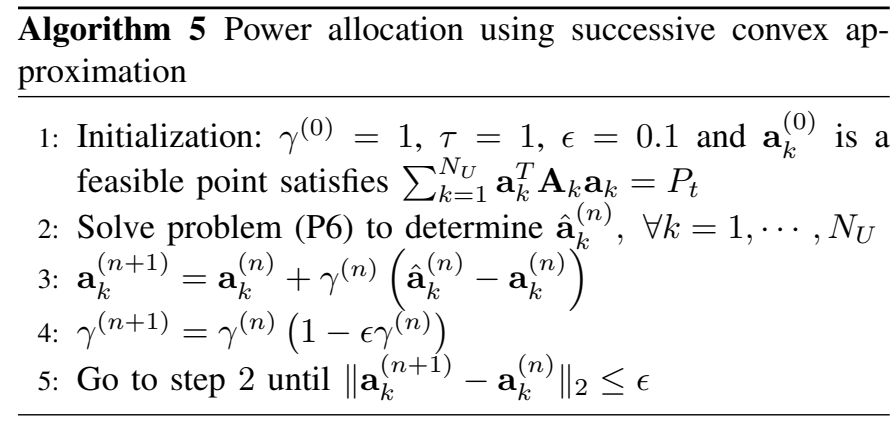

\section{MU power allocation using successive convex approxima-} tion

In order to apply the framework of the successive convex approximation [26], the constrains of the power allocation problem must represent convex set. Thus, we modify the system model in Eq. (47) by replacing the power allocation variables $b_{k}$ and the spatial levels $0 \leqslant \alpha_{0, k} \leqslant 1$ with high level amplitudes $a_{h, k}$ and low level amplitudes $a_{l, k}$ as

$$
\begin{aligned}
\mathbf{x}_{t}^{\mathrm{SCA}} & =\sum_{k=1}^{N_{u}} \mathbf{P}_{k} \mathbf{s}_{i, k} x_{j, k}, s_{i, k}(n) \in\left\{a_{l, k}, a_{h, k}\right\}, \\
\mathrm{E}\left[\mathbf{x}_{t}^{H} \mathbf{x}_{t}\right] & =\sum_{k=1}^{N_{U}} \operatorname{Tr}\left\{\mathbf{P}_{k} \mathbf{R}_{s_{k}, s_{k}} \mathbf{P}_{k}^{H}\right\}=\sum_{k=1}^{N_{U}} \mathbf{a}_{k}^{T} \mathbf{A}_{k} \mathbf{a}_{k}=P_{t}, \\
\mathbf{A}_{k} & =\left[\begin{array}{cc}
c_{k} & d_{k} / 2 \\
d_{k} / 2 & c_{k}
\end{array}\right], \quad \mathbf{a}_{k}=\left[\begin{array}{ll}
a_{l, k} & a_{h, k}
\end{array}\right]^{T}, \\
c_{k} & =\frac{1}{4} \operatorname{Tr}\left\{\mathbf{P}_{k}^{H} \mathbf{P}_{k}\right\}+\frac{1}{4} \operatorname{Tr}\left\{\mathbf{1}_{N_{a, k}} \mathbf{P}_{k}^{H} \mathbf{P}_{k}\right\}, \\
d_{k} & =\frac{1}{2} \operatorname{Tr}\left\{\mathbf{1}_{N_{a, k}} \mathbf{P}_{k}^{H} \mathbf{P}_{k}\right\}-\frac{1}{2} \operatorname{Tr}\left\{\mathbf{P}_{k}^{H} \mathbf{P}_{k}\right\} .
\end{aligned}
$$

Accordingly, the received signal and the combined SNR of the $k^{\text {th }}$ user can be expressed as

$$
\begin{aligned}
\mathbf{y}_{k}^{\text {SCA }} & =\mathbf{s}_{i, k} x_{j, k}+\mathbf{n}_{k}, \\
\mathrm{SNR}_{i, k}^{\mathrm{SCA}} & =\frac{\left(n_{1, i k} a_{h, k}+n_{0, i k} a_{l, k}\right)^{2}}{\left(n_{1, i k}+n_{0, i k}\right) \sigma^{2}} .
\end{aligned}
$$

where $n_{1, i k}$ and $n_{0, i k}$ denote number of ones and zeros in $\mathbf{s}_{i, k}$, respectively. The mutual information of the spatial $\left(I_{S, k}^{\mathrm{SCA}}\right)$ and the modulation $\left(I_{M, k}^{\mathrm{SCA}}\right)$ symbols can be expressed in a similar way to that in Eq. (50). The high and low amplitude levels are designed to maximize the weighted sum $\mathrm{SE}$ as

$$
\text { (P5) }\left\{\begin{array}{l}
\underset{\mathbf{a}_{1}, \cdots, \mathbf{a}_{N_{U}}}{\operatorname{maximize}} U\left(\mathbf{a}_{1}, \cdots, \mathbf{a}_{N_{U}}\right)=\sum_{k=1}^{N_{U}} \rho_{k}\left(I_{M, k}^{\mathrm{SCA}}+I_{S, k}^{\mathrm{SCA}}\right) \\
\text { subject to } \sum_{k=1}^{N_{U}} \mathbf{a}_{k}^{T} \mathbf{A}_{k} \mathbf{a}_{k} \leqslant P_{t} .
\end{array}\right.
$$

In Algorithm 5, we illustrate how the successive convex approximation method solves problem (P5). First, the objective function is approximated using second order expansion at any feasible point as

$$
\left\{\begin{array}{c}
\underset{\mathbf{a}_{1}, \cdots, \mathbf{a}_{N_{U}}}{\operatorname{maximize}} \sum_{k=1}^{N_{U}} \nabla \mathbf{a}_{k}^{n} U(\mathbf{a})_{\left.\right|_{\mathbf{a}=\mathbf{a}^{n}} ^{T}}^{T}\left(\mathbf{a}_{k}-\mathbf{a}_{k}^{n}\right)+ \\
\frac{\tau}{2}\left\|\mathbf{a}_{k}-\mathbf{a}_{k}^{n}\right\|_{2}^{2} \\
\text { subject to } \sum_{k=1}^{N_{U}} \mathbf{a}_{k}^{T} \mathbf{A}_{k} \mathbf{a}_{k} \leqslant P_{t} .
\end{array}\right.
$$

and hence, we solve the convex problem (P6) to obtain $\hat{\mathbf{a}}_{k}^{(n)}$ as shown in step 2 of Algorithm 5. At step 3, we use the determined $\hat{\mathbf{a}}_{k}^{(n)}$ to update the solution $\mathbf{a}_{k}^{(n+1)}$. The above procedure is repeated until convergence.

\section{Simulation Results}

In this section, we evaluate the system performance of the proposed RSM schemes compared to CM methods for single and multiple users transmissions. In the simulations environment, we consider the stochastic channel model in Eq. (7) with $g_{i} \sim \mathcal{C N}(0,1),\left(\phi_{i} \in[-\pi / 6, \pi / 6], \theta_{i} \in[-\pi, \pi]\right)$ are uniformly distributed and $\sigma^{2}=-84 \mathrm{dBm}$. The proposed UT architecture in Fig. 1c is flexible where it works either with RSM or CM transmissions through adjusting controlling switches. Thus, flexible RSM (FRSM) scheme achieves the best performance of RSM and CM methods.

Fig. 2 shows the SE comparison between RSM-DC, RSMIC and CM schemes for single user system assuming single RF chain at the UT and the RAS method proposed in Algorithm 1. At low number of scattering clusters $C$, the IC scheme outperforms the DC but they have similar performance at high $C$. In the DC scheme, the modulation symbol is not transmitted with high probability at low $C$ and this degrades the SE. Higher values of $C$ allow transmitting more spatial bits and thus, the SE of the RSM schemes improve more than CM transmission considering single RF at the UT.

Fig. 3 illustrates the SE of the FRSM scheme compared to the CM transmission for single user system assuming multiple RF chains at the UT and applying the RAS procedure proposed in Algorithm 1. The FRSM scheme outperforms the CM especially at low number of RF chains at the UT. Increasing $N_{r f}$ at the UT allows transmitting more modulation symbols and single spatial symbol. Thus, the SE of FRSM and CM transmissions are approaching at high $N_{r f}$.

Fig. 4 shows the $\mathrm{SE}-\mathrm{EE}_{\mathrm{UT}}$ trade-off of the FRSM scheme compared to $\mathrm{CM}$ transmission for the single user system assuming multiple RF chains at the UT and the RAS method in Algorithm 1. The FRSM outperforms the CM in SE and EE at low and high SNR regimes. Increasing $N_{r f}$ improves the SE and increases the power consumption at the UT which entails a degradation in the $\mathrm{EE}_{\mathrm{UT}}$.

Fig. 5 illustrates the $\mathrm{SE}-\mathrm{EE}_{\mathrm{BS}}$ trade-off of the proposed FRSM scheme with RAS compared to that with RAS and TAS for the single user system. Applying TAS after RAS improves the $\mathrm{EE}_{\mathrm{BS}}$ as the power consumed by the RF chains at the BS reduces. However, TAS entails a reduction in the $\mathrm{SE}$ as deactivating the transmit antennas reduces the transmit beamforming gain.

Fig. 6 shows the SE of the proposed FRSM scheme with the RAS and TAS methods proposed in Algorithms 1 and 2; respectively, compared to that of exhaustive search based antenna selection. The proposed antenna selection algorithms approach the performance of the exhaustive search with a significant reduction in the computational complexity as illustrated in Eq. (45).

Fig. 7 illustrates the sum SE of four users by allocating the power using the alternating optimization method proposed in 


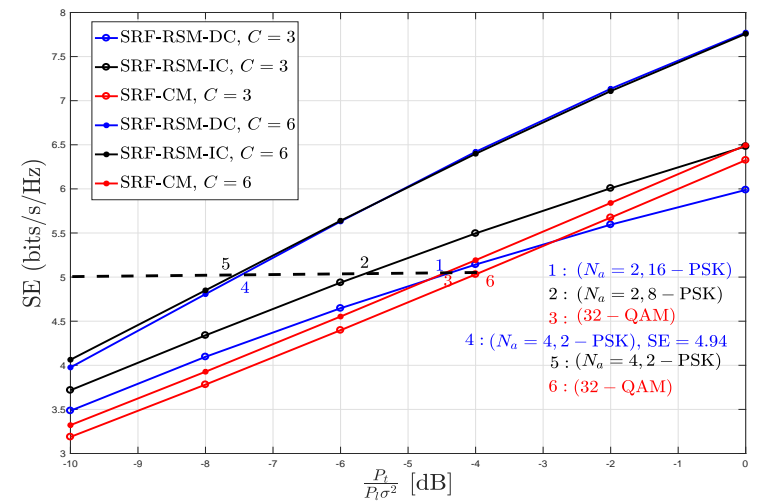

Fig. 2: Spectral efficiency of the single user RSM-DC, RSMIC and CM assuming single RF UT, $N_{t}=128, N_{r}=16$ and (overage over 1000 channel realizations).

Algorithm 4 compared to that of successive convex approximation illustrated in Algorithm 5 assuming ten random initial points. The proposed alternating optimization converges much faster than the successive convex approximation.

Fig. 8 shows the per user spatial and modulation symbols mutual information. We allocate the power among four users by applying the proposed alternating optimization algorithm. Users with high SNR, achieve higher values of modulation symbol mutual information while the spatial mutual information saturates at low SNR as we transmit spatial bits as many as number of active antennas at the UT (less than or equal to C).

Fig. 9 shows the sum SE of the proposed joint user, antenna and power allocation Algorithm 3 compared to that of exhaustive search based antenna selection over all the users. The proposed algorithm tightly approaches the performance of the exhaustive search but with significant reduction in the search complexity.

Fig. 10 illustrates the sum SE of the proposed RSM scheme in Algorithm 3 with alternating optimization power allocation compared to $\mathrm{CM}$ transmission with water filling power allocation [39]. The RSM scheme achieves higher values of SE than $\mathrm{CM}$ as the spatial mutual information requires low SNR to reach saturation as depicted in Fig. 8.

\section{CONCLUSION}

In this paper, we proposed RSM scheme for the DL of multi-user MIMO system operating in mmWave outdoor and narrowband environment. We developed novel and energy efficient flexible UT architecture that works with RSM or CM transmissions. The spatial mutual information saturates at low SNR and thus, RSM schemes outperforms CM especially at low SNR. We extended the work in [21] into multiple RF chains at the UT and we studied the SE-EE trade-off. We proposed receive and transmit antenna selection algorithms aimed at maximizing the BS energy efficiency. These algorithms approach the exhaustive search performance but with much less computational complexity. For the multi-user scenario, we jointly optimized number of users, set of antennas and the

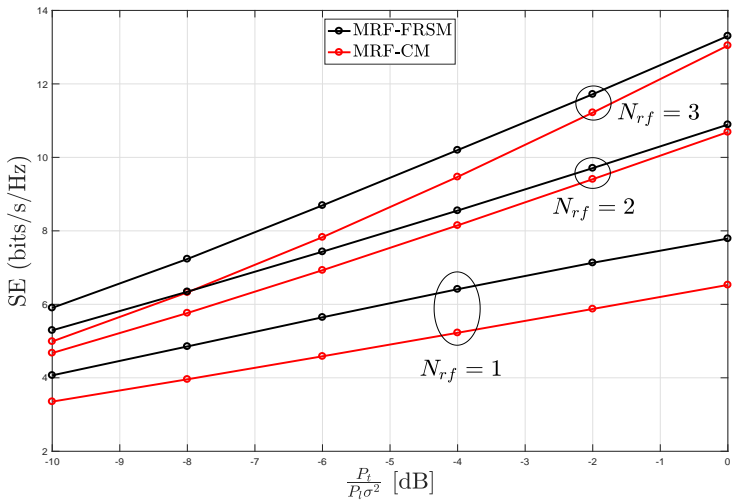

Fig. 3: Spectral efficiency of the single user flexible RSM compared to CM assuming multiple RF UT, $N_{t}=128, N_{r}=$ $16, C=6$ and (overage over 1000 channel realizations).

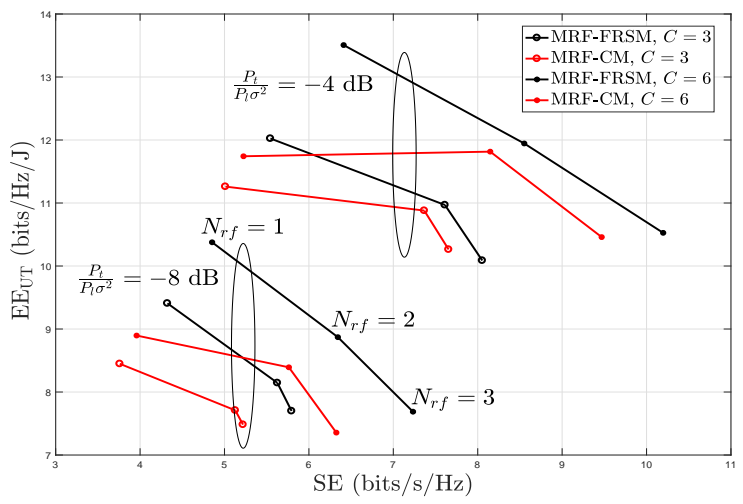

Fig. 4: Spectral-energy efficiency at the UT trade-off of the single user flexible RSM compared to CM assuming multiple RF UT, $N_{t}=128, N_{r}=16$ and (overage over 1000 channel realizations).

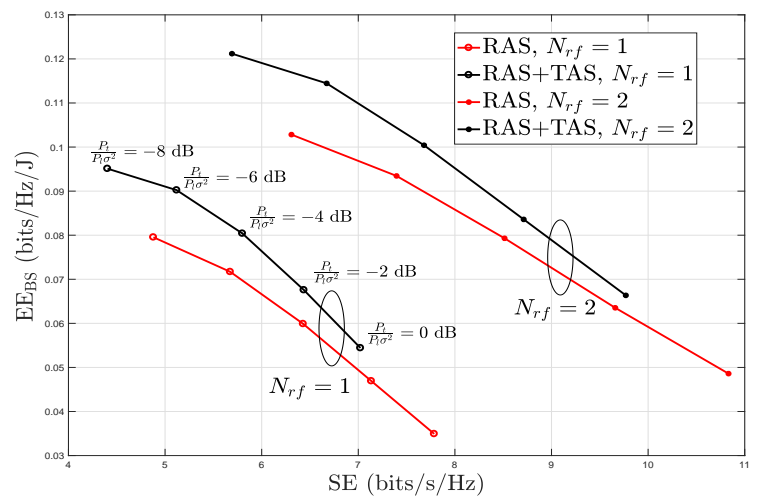

Fig. 5: Spectral-energy efficiency at the BS trade-off of the single user flexible RSM with RAS and with RAS+TAS at $N_{t}=128, N_{r}=16, C=6$ and (overage over 1000 channel realizations).

power allocated per user to maximize the weighted sum SE. At given set of users and antenna per user, we formulate the power 


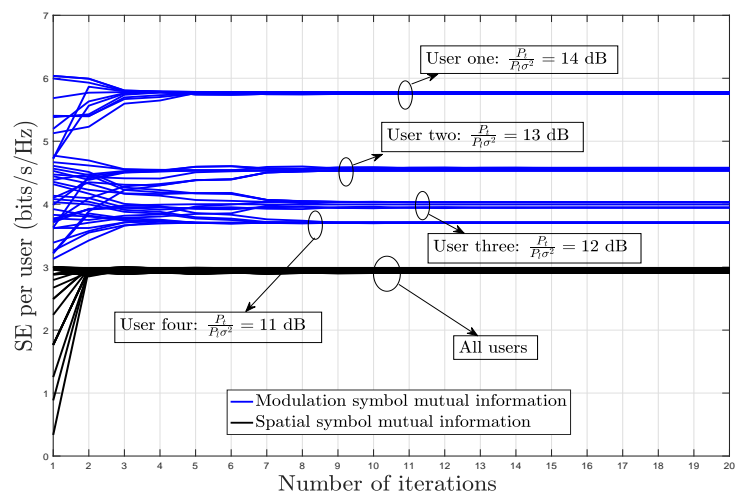

Fig. 8: Per user spatial and modulation symbols mutual information of the proposed alternating optimization scheme assuming 10 random initial points, $N_{U}=4, C=3$, $P_{t} / P_{l} \sigma^{2}=14,13,12$ and $11 \mathrm{~dB}$ for the four users, $N_{t}=128$, $N_{r}=16$ and (overage over 1000 channel realizations).

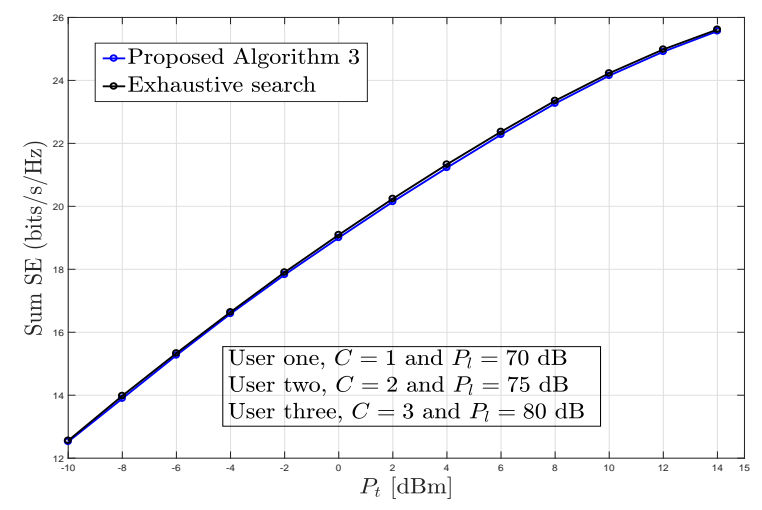

Fig. 9: Sum SE of the proposed joint user, antenna selection and power allocation Algorithm 3 compared to the exhaustive search over all the UTs antennas combinations at $N_{U}=3$, $C=1,2,3$ and $P_{l}=70,75,80 \mathrm{~dB}, N_{t}=128, N_{r}=3$ and (overage over 1000 channel realizations).

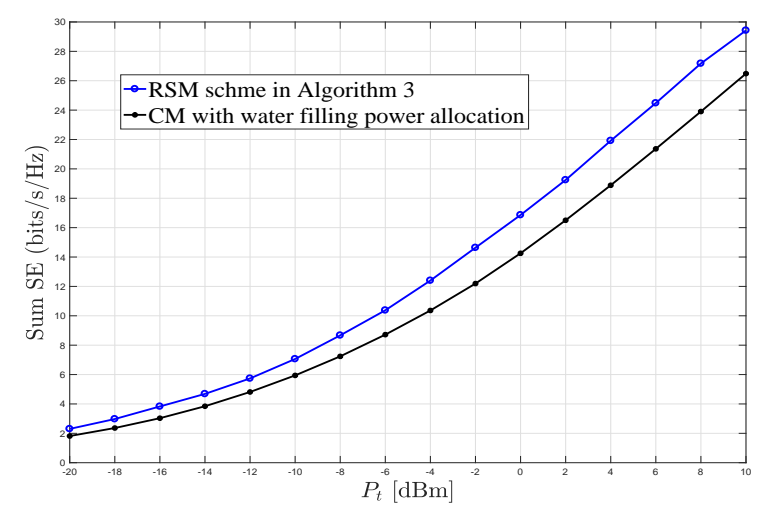

Fig. 10: Sum SE of the proposed RSM in Algorithm 3 compared to CM with water filling power allocation at $N_{U}=4$, $N_{r f}=1, C=6$ and $P_{l}=80,85,90,95 \mathrm{~dB}, N_{t}=128$, $N_{r}=16$ and (overage over 1000 channel realizations).

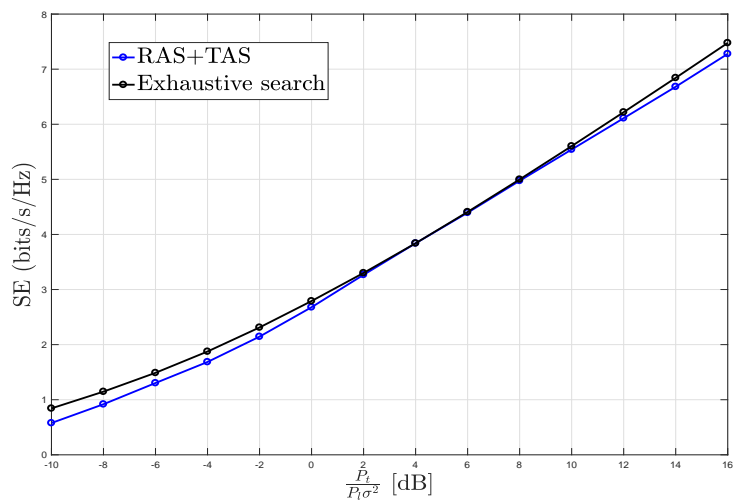

Fig. 6: Spectral efficiency of the single user flexible RSM with the proposed RAS+TAS algorithms compared to that with exhaustive search based antenna selection at $N_{r f}=1, N_{t}=8$, $N_{r}=4, C=3$ and (overage over 1000 channel realizations).

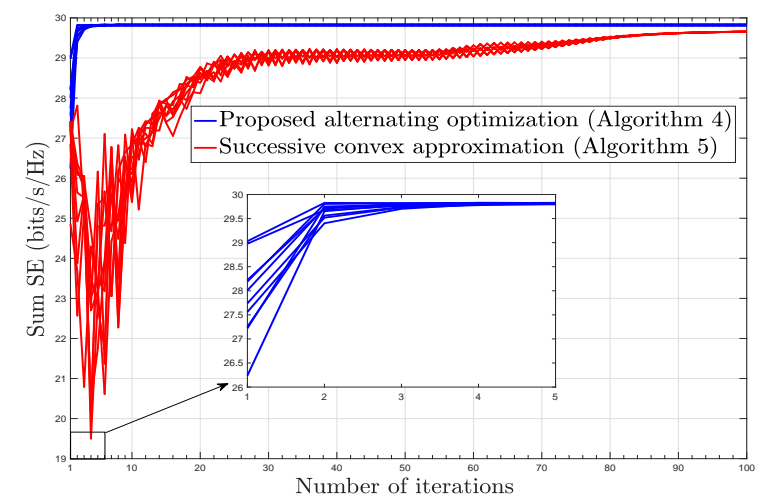

Fig. 7: Sum SE of the proposed alternating optimization scheme compared to the successive convex approximation technique assuming 10 random initial points, $N_{U}=4, C=3$, $P_{t} / P_{l} \sigma^{2}=14,13,12$ and $11 \mathrm{~dB}$ for the four users, $N_{t}=128$, $N_{r}=16$ and (overage over 1000 channel realizations).

allocation as non convex optimization problem. Then, we proposed alternating optimization algorithm to convert the non convex program into many convex problems. The proposed alternating optimization outperforms the successive convex approximation in terms of performance and convergence. The study of the uplink multiple access channel using TSM is a future work topic.

\section{REFERENCES}

[1] International Telecommunication Union (ITU). IMT traffic estimates for the years 2020 to 2030, Report ITU-R M.2370-0, Jul. 2015.

[2] L. Ericsson, "More than 50 billion connected devices," White Paper, vol. 14, p. 124, Feb. 2011.

[3] T. S. Rappaport et al., "Millimeter wave mobile communications for 5G cellular: It will work!" IEEE access, vol. 1, pp. 335-349, May 2013.

[4] F. Boccardi et al., "Five disruptive technology directions for 5G," IEEE Communications Magazine, vol. 52, no. 2, pp. 74-80, Feb. 2014.

[5] W. Roh et al., "Millimeter-wave beamforming as an enabling technology for $5 \mathrm{G}$ cellular communications: Theoretical feasibility and prototype results," IEEE communications magazine, vol. 52, no. 2, pp. 106-113, Feb. 2014. 
[6] A. I. Sulyman et al., "Radio propagation path loss models for $5 \mathrm{~g}$ cellular networks in the $28 \mathrm{ghz}$ and $38 \mathrm{ghz}$ millimeter-wave bands," IEEE Communications Magazine, vol. 52, no. 9, pp. 78-86, Sep. 2014.

[7] J. G. Andrews et al., "What will 5G be?" IEEE Journal on selected areas in communications, vol. 32, no. 6, pp. 1065-1082, Jun. 2014.

[8] A. Alkhateeb et al., "Channel estimation and hybrid precoding for millimeter wave cellular systems," IEEE Journal of Selected Topics in Signal Processing, vol. 8, no. 5, pp. 831-846, Oct. 2014.

[9] O. El Ayach et al., "Spatially sparse precoding in millimeter wave MIMO systems," IEEE transactions on wireless communications, vol. 13, no. 3, pp. 1499-1513, Mar. 2014.

[10] R. W. Heath et al., "An overview of signal processing techniques for millimeter wave MIMO systems," IEEE journal of selected topics in signal processing, vol. 10, no. 3, pp. 436-453, Apr. 2016.

[11] R. Méndez-Rial et al., "Hybrid MIMO architectures for millimeter wave communications: Phase shifters or switches?" IEEE Access, vol. 4, pp. 247-267, Jan. 2016.

[12] X. Gao et al., "Energy-efficient hybrid analog and digital precoding for mmwave MIMO systems with large antenna arrays," IEEE Journal on Selected Areas in Communications, vol. 34, no. 4, pp. 998-1009, Apr. 2016.

[13] X. Yu et al., "Alternating minimization algorithms for hybrid precoding in millimeter wave MIMO systems," IEEE Journal of Selected Topics in Signal Processing, vol. 10, no. 3, pp. 485-500, Apr. 2016.

[14] S. He et al., "Energy-efficient transceiver design for hybrid sub-array architecture MIMO systems," IEEE Access, vol. 4, pp. 9895-9905, 2016.

[15] R. Y. Mesleh et al., "Spatial modulation," IEEE Transactions on vehicular technology, vol. 57, no. 4, pp. 2228-2241, Jul. 2008.

[16] J. Jeganathan, A. Ghrayeb, and L. Szczecinski, "Spatial modulation: Optimal detection and performance analysis.," IEEE Communications Letters, vol. 12, no. 8, pp. 545-547, Aug. 2008.

[17] M. Di Renzo, H. Haas, and P. Grant, "Spatial modulation for multipleantenna wireless systems: A survey," IEEE Communications Magazine, vol. 49, no. 12, pp. 182-191, Dec. 2011.

[18] A. Younis et al., "Generalised spatial modulation," in the Forty Fourth Asilomar Conference on Signals, Systems and Computers, pp. 14981502, Nov. 2010.

[19] M. D. Renzo, "Spatial modulation for generalized MIMO: Challenges, opportunities and implementation," Proceedings of the IEEE, vol. 102, no. 1, pp. 56-103, Jan. 2014.

[20] N. S. Perovic, P. Liu, M. Di Renzo, and A. Springer, "Receive spatial modulation for los mmwave communications based on TX beamforming," IEEE Communications Letters, Dec. 2016.

[21] A. Raafat, A. Agustin, and J. Vidal, "Receive spatial modulation for massive MIMO systems," in IEEE Global Communications Conference (GLOBECOM), pp. 1-6, Dec. 2017.

[22] _ "Mmse precoding for receive spatial modulation in large MIMO systems," in 19th IEEE International Workshop on Signal Processing Advances in Wireless Communications (SPAWC), pp. 1-5, Jun. 2018.

[23] A. Raafat, A. Agustin, and J. Vidal, "Wideband receive spatial modulation with time domain pre-equalizer for large MIMO systems," in IEEE Globecom Workshops (GC Wkshps), pp. 1-7, 2018-12.
[24] A. Duarte and R. Sampaio-Neto, "Precoding and spatial modulation in the downlink of MU-MIMO systems," arXiv preprint arXiv:1803.01895, 2018.

[25] G. Scutari, F. Facchinei, and L. Lampariello, "Parallel and distributed methods for constrained nonconvex optimization-Part I:Theory," IEEE Transactions on Signal Processing, vol. 65, no. 8, pp. 1929-1944, Apr. 2016.

[26] G. Scutari et al., "Decomposition by partial linearization: Parallel optimization of multi-agent systems," IEEE Transactions on Signal Processing, vol. 62, no. 3, pp. 641-656, Feb. 2013.

[27] S. Rami, W. Tuni, and W. R. Eisenstadt, "Millimeter wave MOSFET amplitude detector," Topical Meeting on Silicon Monolithic Integrated Circuits in RF Systems (SiRF), pp. 84-87, Jan. 2010.

[28] A. Raafat, A. Agustin, and J. Vidal, "Receive antenna selection and hybrid precoding for receive spatial modulation in massive MIMO systems," in the 22nd International ITG Workshop on Smart Antennas (WSA), pp. 1-8, Mar. 2018.

[29] S. Shakib et al., "A highly efficient and linear power amplifier for 28-GHz 5G phased array radios in 28-nm CMOS," IEEE Journal of Solid-State Circuit, vol. 51, no. 12, pp. 3020-3036, Dec. 2016.

[30] J. Lagos et al., "A single-channel, 600-MS/s, 12-b, ringamp-based pipelined ADC in 28-nm cmos," IEEE Journal of Solid-State Circuits, vol. 54, no. 2, pp. 403-416, Feb. 2018

[31] N. Rostomyan, M. Özen, and P. Asbeck, "28 GHz doherty power amplifier in cmos soi with $28 \%$ back-off pae," IEEE Microwave and Wireless Components Letters, vol. 28, no. 5, pp. 446-448, May 2018.

[32] M. R. Akdeniz et al., "Millimeter wave channel modeling and cellular capacity evaluation," IEEE Journal on Selected Areas in Communications, vol. 32, no. 6, pp. 1164-1179, Jun. 2014.

[33] M. K. Simon, Probability distributions involving Gaussian random variables: A handbook for engineers and scientists. Springer, May 2007.

[34] F. Chapeau-Blondeau, "Noise-enhanced capacity via stochastic resonance in an asymmetric binary channel," Physical Review E, vol. 55, no. 2, p. 2016, Feb. 1997.

[35] J. M. Geist, "Capacity and cutoff rate for dense M-ary PSK constellations," in IEEE Conference on Military Communications, pp. 768-770, Sep. 1990.

[36] P. B. Stark and R. L. Parker, "Bounded-variable least-squares: An algorithm and applications," Computational Statistics, vol. 10, pp. 129-129, 1995.

[37] G. D. Forney and G. Ungerboeck, "Modulation and coding for linear Gaussian channels," IEEE Transactions on Information Theory, vol. 44, no. 6, pp. 2384-2415, Oct. 1998.

[38] Y. Wu et al., "Receive antenna selection in the downlink of multiuser MIMO systems," 62nd Vehicular Technology Conference, vol. 1, pp. 477-481, Sep. 2005.

[39] N. Jindal, S. Jafar, S. Vishwanath, and A. Goldsmith, "Sum power iterative water-filling for multi-antenna Gaussian broadcast channels," in the 36th Asilomar Conference on Signals, Systems and Computers., vol. 2, pp. 1518-1522, Nov. 2002. 